\title{
Insulin, Diabetes, and the Cell Membrane: An Hypothesis
}

\author{
D. E. McMillan \\ Sansum Medical Research Foundation, Santa Barbara, California, USA
}

Bryszewska and Leyko [1] report in this issue a further thread in the developing fabric of observations that suggests a novel mechanism for hormone action. The new concept is useful to many investigators, linking currently separate diabetes research areas. The loom on which this conceptual fabric is being woven is the cell membrane. Our understanding of this structure has risen rapidly in the last few years.

The erythrocyte supplies us with the simplest and most easily available human cell plasma membrane. For this reason it has been studied more extensively than any other. Erythrocyte flow behaviour is known to be altered in diabetes [2], a disturbance rapidly reversed by intravenous insulin administration [3]. 'Diabetic' erythrocytes form doublets both less regularly and more slowly than normal erythrocytes [4]. The reason for slowing of doublet formation has been analyzed [Microvascular Research, in press]. 'Diabetic' erythrocytes are not truly stiffened; instead, the erythrocyte membrane is at least twice as resistant to rate of change of curvature as the normal erythrocyte membrane. What might insulin do to the cell surface that so improves this unusual membrane property?

Reports of red cell membrane abnormalities in diabetes suggest a basis. A 3.4\% increase in 'microviscosity' of erythrocyte membrane ghosts in diabetes has been reported [5]. The 'microviscosity' finding is based on a slowed rate of movement of membrane-seeking fluorescent compounds. In diabetes the molecules activated by polarized ultraviolet light change position more slowly, emitting visible light photons closer in orientation to the original exciting photon. In Bryszewska and Leyko's study fluorescence excitation is also used, although the conditions and meaning are different. Pyrene is the fluorescence-susceptible molecule used. Post-excitation behaviour is linked not to changing monomer position but to pyrene's self-association tendency. A single excited pyrene molecule emits a photon of $396 \mathrm{~nm}$ wavelength, but when two pyrene molecules combine, the excited dimer (excimer) emits a $470 \mathrm{~nm}$ (lower energy) photon. Bryszewska and Leyko have found decreased excimer radiation from the 'diabetic' erythrocyte membrane. Now they report that this decrease in excimer level is reversed by incubation in insulin at a nearly physiological level, $10^{-9} \mathrm{~mol} / 1$. The corrective effect, though not quantitatively striking (about $4.7 \%$ ), occurs consistently. Both studies suggest that insulin affects membrane lipid properties.

What kind of change is taking place? Excimer formation is known to be affected by membrane temperature [6] and applied pressure [7]. The temperature effect is complex. At low temperature, lipid bilayers exhibit solid properties and are considered to be in a gel form; excimer radiation falls with temperature. At levels above a specific temperature for each type of membrane lipid combination, lipid bilayers exhibit non-solid behaviour. During the solid to non-solid conversion the membrane becomes thinner by more than $20 \%$, each phospholipid molecule substantially expanding the surface area occupied. Biologically active membranes are characteristically non-solid. The non-solid membrane has both solid and fluid attributes so that it is referred to as liquid crystal in state. The excimer to monomer ratio falls as the membrane develops liquid crystal behaviour and then rises again as continuing temperature increase causes the membrane lipids to be more cohesive, allowing the pyrene molecules in the bilayer to form dimers. The 'diabetic' erythrocyte membrane acts as though it is at a different temperature or higher pressure than the normal membrane.

Both the reported in vitro effect of insulin on erythrocyte deformability [3] and the change in excimer ratio [1] are especially remarkable because insulin has an effect when so few insulin receptor sites are present on the erythrocyte cell membrane. There is other in vitro evidence that insulin affects the behaviour of erythrocyte membranes. Several years ago, Dormandy and Zarday showed that erythrocyte oxidation-reduction transport, $\mathrm{pH}$ gradient generation, and ion transport are altered by the presence of insulin levels in a dose response matching its physiological range [9]. This insulin effect has been linked to the migration of NAD in its reduced 
form across the cell membrane [10,11]. The erythrocyte also appears to respond to parathyroid hormone [12]. The mature erythrocyte membrane usually contains less than 100 insulin receptors [8]. Since its surface area is approximately 140 square micrometers the receptors are probably separated from each other by 1 micrometer or more, a rather striking distance for insulin-receptor complexes to influence the intervening membrane lipids. When so few receptors are required and both mitochondria and a cell nucleus are absent, a new implication about hormone action may be drawn. Insulin could well have an effect on the erythrocyte and other tissues by modifying the physical state of the cell membrane, either the lipid bilayer or the lipid-protein complex.

The erythrocyte plasma membrane is $55 \%$ protein and $45 \%$ lipid. Phospholipid and unesterified cholesterol are present in nearly molecularly equal amounts $(0.8-1.0)$ in the lipid fraction. Types of phospholipid are distributed differently in the two bilayer halves. Phosphatidylcholine and sphingomyelin dominate the outer layer while almost all the phosphatidylethanolamine, phosphatidylserine, and phosphatidylinositol are present in the inner (haemoglobin-facing) layer. The protein components of the erythrocyte membrane are divided into those that are embedded in the lipid layer, the integral proteins, and those that are not. The integral proteins are dominated by band 3 [13] and include the cytochalasin B-binding hexose transport protein [14]. The non-integral proteins are divided into a major internal set and a minor external set. The inner surface proteins form a network that is responsible for the erythrocyte's ability to return to its discoid shape after deformation. The outer surface contains the glycoproteins responsible for blood group antigenicity.

How can we picture the effect of hormones on the behaviour of the thin lipid films that bind the cell? Three fairly simple models are available to assist us. The first model, the liquid crystal display, surrounds us more and more each passing day. It forms the faces of our watches, our calculators, and many other word and number display devices. Liquid crystal displays have been selected for use because they require very little electrical energy to maintain locally altered reflectivity. Their existence shows that it is possible, with a very small commitment of force, to alter strikingly the organization of a material that resembles biological membrane lipid. The secret of the liquid crystal is the presence of some order in a material that otherwise has the characteristics of a fluid [15]. The electrical field applied to the liquid crystal display changes the magnitude of this order, modifying the substance's optical properties. It is attractive to consider that insulin and other surfacebound hormones have a similar ordering effect on the plasma membrane.

The second model comes from the study of an unusual substance called a black film. Black films are bilayer lipid membranes formed in a small cylinder, com- posed of two organic compounds not necessarily derived from living tissues. Under appropriate conditions a thin, optically unrecognizable (black) membrane forms after a drop of an appropriate lipid mixture is placed across the circular hole that forms the cylinder. This membrane resists the movement of water so that pressure can be applied across it and its surface tension measured [16]. Black films characteristically have very low bilayer surface tensions ( $<5$ dynes/cm or ergs/ $\mathrm{cm}^{2}$ ). Surface tension is equivalent to the free energy required to enlarge the surface. The surface tension of erythrocyte membranes has been measured recently by a less direct technique than that used to study black films. Two polymer-containing physiological solutions with slightly different surface tensions were used to detect an extremely low external surface tension $(6.5 \times$ $10^{-4}$ dynes $/ \mathrm{cm}$ ) [17]. Water has a surface tension of about 70 dynes $/ \mathrm{cm}$. This very low surface tension is linked to the unusual flexibility of red cell membranes. Insulin-receptor interaction might affect the surface tension (free energy) of the cell membrane.

The third illustration of lipid bilayer behaviour comes from the study of liposomes, multilayered phospholipid vesicles that are often used to study membrane bilayer behaviour. Because liposomes can easily be made in bulk they have been used not only to study membrane thermal transitions and permeability but also to examine resistance of lipid bilayers to fusion. Fusion is the process by which two bilayers join each other, such as when intracellular vesicles attach to the internal surface of the B cell plasma membrane during insulin release. Membrane fusion occurs only when the substantial resistance barrier present on both bilayer surfaces breaks down. The high resistance is due to local Zwitterion effects called hydration forces. A hydrostatic pressure of $7 \times 10^{9}$ dynes $/ \mathrm{cm}^{2}(1,000$ atmospheres) generated by these forces at the bilayer surface falls off very rapidly to an inconsequential level 50 Angstroms away [18]. This pressure, linked to organization of water near the surface, is strikingly altered by such old biological friends as calcium and magnesium. The effect of these cations is influenced by local concentrations of specific phospholipids. Phosphatidylethanolamine favours fusion if it replaces phosphatidylcholine. Insulin-receptor interaction might affect phospholipid distribution or the magnitude of the surface pressure.

These three examples furnish us with speculations about possible mechanisms of hormone action, but clear understanding must await the progress in membrane research that will occur in this decade. The actual mechanisms by which hormones influence the cell membrane's conduct now appear discernible. The recently reported [19] surface appearance of previously sequestered hexose transport activity following insulin incubation may be linked to membrane physical change. There is also an association between insulin, hyperglycaemia, and membrane myoinositol turnover. The phosphatidylinositol present in the inner plasma 
membrane layer is high in arachidonic acid, acting as its source in prostaglandin, prostacyclin and thromboxane synthesis. This reservoir is disrupted in diabetes, a change associated with low free intracellular myoinositol [20]. In diabetes, declining free intracellular myoinositol blocks arachidonic acid storage affecting prostaglandin metabolism [21]. Insulin's influence is less clearly direct here, but an effect through altered cell membrane organization is plausible.

The lessons learned from insulin's ability to affect the behaviour of the lowly red blood cell should have wide applicability. The erythrocyte is not only a source of easily studied cell membranes, free of mitochondria and a cell nucleus; it is also a cell designed to participate in blood flow, a physical process subtly altered by the diabetic state. Insulin's effect on this process now suggests an entirely new mode of hormone action, alteration of the physical state of the cell plasma membrane.

\section{References}

1. Bryszewska M, Leyko W (1983) In vitro effect of insulin on lateral mobility of fluorescent probe in human erythrocyte membrane in diabetes mellitus. Diabetologia 24: 311-313

2. McMillan DE, Utterback NG, La Puma J (1978) Reduced erythrocyte deformability in diabetes. Diabetes 27: 895-901

3. Juhan I, Vague P, Buonocore M, Moulin JP, Calas MF, Vialettes B, Verdot JJ (1981) Effects of insulin on erythrocyte deformability in diabetes-relationship between erythrocyte deformability in platelet aggregation. Scand J Clin Lab Invest 41: 159-164

4. McMillan DE, Utterback NG, Sparks LL, Bramwell PC (1981) Impaired erythrocyte doublet formation in diabetes. Diabetologia 21: $575-578$

5. Baba Y, Motoaki K, Kamada T, Setoyama S, Otsuji S (1979) Higher levels of erythrocyte membrane microviscosity in diabetes. Diabetes $28: 1138-1140$

6. Galla H-J, Sackmann E (1974) Lateral diffusion in the hydrophobic region of membranes: use of pyrene excimers as optical probes. Biochim Biophys Acta 339: 102-115

7. Flamm M, Okubo T, Turro NJ, Schachter D (1982) Pressure dependence of pyrene excimer fluorescence in human erythrocyte membranes. Biochim Biophys Acta 687: 101-104
8. Dons RF, Corash LM, Gorden $\mathbf{P}$ (1981) The insulin receptor is an age-dependent integral component of the human erythrocyte membrane. J Biol Chem 256:2982-2987

9. Dormandy TL, Zarday Z (1965) The mechanism of insulin action: The immediate electrochemical effects of insulin on red cell systems. J Physiol 80: 684-707

10. Low H, Crane FL, Grebing C, Tally M, Hall K (1978) Hormone control of plasma membrane oxidation-reduction reactions. FEBS Letters 91: 166-168

11. Crane FL, Sun IL, Low H, Clark MG (1982) Insulin control of transplasma membrane NADH dehydrogenase. Fed Proc 41: 1339

12. Bogin E, Massry SG, Levi J, Djaldeti M, Bristol G, Smith JP (1982) Effect of parathyroid hormone on osmotic fragility of human erythrocytes. J Clin Invest 69: 1017-1025

13. Knauff PA (1979) Erythrocyte anion exchange and the Band 3 protein: Transport kinetics and molecular structure. In: Bronner F, Kleinzeller A (eds) Current topics in membranes and transport. Academic Press, New York, pp 249-363

14. Czech MP, Massague J, Pilch PF, Carter-Su C (1980) Structural features of the insulin effector system: relation to hexose transport activation. Ann NY Acad Sci 358: 282-291

15. deGennes PE (1974) The physics of liquid crystals. Clarendon Press, Oxford

16. Tien HT (1974) Bilayer lipid membranes: theory and practice. Marcel Dekker, New York

17. Schurch S, Gerson DF, Mclver DJL (1981) Determination of cell/ medium interfacial tensions from contact angles in aqueous polymer systems. Biochim Biophys Acta 640: 557-571

18. Rand RP (1981) Interacting phospholipid bilayers: measured forces and induced structural changes. Ann Rev Biophys Bioeng 10: $277-314$

19. Kono T, Suzuki K, Dansey LE, Robinson FW, Blevins TL (1981) Energy-dependent and protein synthesis-independent recycling of the insulin-sensitive glucose transport mechanism in fat cells. $J$ Biol Chem 256: 6400-6407

20. Lapetina EG, Billah MM, Cuatrecasas P (1981) The phosphatidylinositol cycle and the regulation of arachidonic acid production. Nature 292: 367-369

21. Simmons DA, Winegrad AI, Martin DB (1982) Significance of tissue myo-inositol concentrations in metabolic regulation in nerve. Science 217: 848-851

Dr. D. E. McMillan

Eisenhower Medical Center

Rancho Mirage

California

USA 